\title{
Self-organising interference coordination in optical wireless networks
}

\author{
Birendra Ghimire ${ }^{1 *}$ and Harald Haas ${ }^{1,2}$
}

\begin{abstract}
In this article, self-organising interference management for optical wireless networks deployed inside an aircraft cabin is investigated. A user that has received data in a given frame and intends to continue receiving data in the next frame broadcasts a busy burst (BB) in a time-multiplexed BB slot. The tagged access point (AP) intending to reuse a resource reserved in a neighbouring cell must listen to the BB slot. Provided that channel reciprocity holds, the tagged AP infers (prior to transmission) the amount of co-channel interference (CCl) potentially caused towards the victim user in neighbouring cell. This is a vital information for an AP to decide without any central supervision whether to transmit or defer the transmission to another time or frequency slot so as to limit $\mathrm{CCl}$ caused to the active link to a threshold value. Simulation results demonstrate that the BB approach significantly improves both fairness and spectral efficiency in the system compared to a static resource partitioning approach.
\end{abstract}

\section{Introduction}

Data transmission using optical wireless has been identified as a technology that can be utilised for communications in critical environments, such as aircrafts or hospitals, where radio frequency (RF)-based transmissions are usually prohibited or refrained to avoid interference with critical systems. Moreover, a huge amount of unregulated bandwidth is available at infra-red and visible light frequencies. Likewise, optical wireless signals can be confined within a room which inherently addresses concerns over the eavesdropping of data. In addition, commercially available light emitting diodes (LEDs) and photodiodes (PDs) can be utilised for data transmission and reception. Therefore, there has been a considerable interest in utilising the frequencies at the infra-red and visible spectrum for data transmission [1-7].

The state-of-the-art technique [7] that utilises on-off keying (OOK) for transmitting data using visible light is rather inflexible when it comes to sharing bandwidth among multiple competing users served by an AP that have variable rate requirements. The above shortcoming is addressed by utilising optical intensity modulation (IM)/direct detection (DD) orthogonal frequency

\footnotetext{
* Correspondence: contact@birendra.com

'School of Engineering and Science, Jacobs University Bremen, 28759, Bremen, Germany

Full list of author information is available at the end of the article
}

division multiplexing (OFDM) for data communication. Using OFDM technique [8,9], the available bandwidth can be shared among multiple users by assigning each user a different amount of bandwidth corresponding to the user demand and the scheduling policies. Likewise, link adaptation can be carried out to scale the user throughput according to the prevalent channel conditions at the receiver. The composite baseband signal is modulated onto the optical carrier by varying the optical power proportional to the baseband signal amplitude in an optical IM/DD system. The fluctuation in instantaneous optical power is detected by a PD, which converts the received optical signal to an electrical signal, which can be decoded by the receiver. Even if LEDs that radiate in the visible light spectrum are used, the fluctuations in optical power are imperceptible to human eye but can be easily detected by a PD. As such, the LEDs can be used simultaneously for both lighting and data transmission.

Although the overall bandwidth in the visible or infrared spectrum is in the tetrahertz $(\mathrm{THz})$ range, the bandwidth of the signal that can be utilised by an optical IM/DD system using LEDs is inherently limited by the bandwidths of transmitter and receiver frontends. In order to serve multiple users and have ubiquitous system coverage, it becomes necessary to reuse the available bandwidth. Although bandwidth reuse potentially increases the system capacity, transmission of data 
intended for a user inevitably causes interference towards other users receiving data on the same resource and vice versa. An orthogonal frequency division multiple access (OFDMA) network where the chunks (timefrequency slots) are fully reused is prone to high CCI at cell-edge. This potentially causes a significant reduction of user throughout and causes outage at the cell-edge in the worst case. Therefore, interference coordination is essential among multiple optical cells within the network in order to balance system capacity against enhanced throughput at the cell-edge.

To achieve the aforesaid goal, several interference coordination mechanisms have been investigated in the literature. Static resource partitioning $[10,11]$ using traditional cluster based frequency planning approach is the most commonly used approach. With this approach, the users served by a tagged AP are restricted to use a certain fixed subset of the available chunks in the system. CCI is mitigated by ensuring that any two cells that reuse the same set of chunks are separated in space by a minimum reuse distance. The shortcoming of such approach is that it can cause some of the chunks to remain idle even though transmitting data on these chunks would not cause detrimental CCI towards other users in the neighbouring cells. Furthermore, if the instantaneous traffic loads varies widely among cells within the network [12], such approach can lead to wastage of resource in lightly loaded cells and fail to cater for the traffic demands in heavily loaded cells. The available system bandwidth and consequently the share of bandwidth per user can be enhanced by using the principle of wavelength division multiplexing (WDM) [13] such that a tagged cell can use one of the three primary colours, i.e. red, green and blue, for data transmission which retains the advantage of the frequency planning approach and at the same time enhances the system bandwidth. However, such approach adds to the cost of receiver unit because separate filters and PD with peak spectral response for each colour band are required.

An entirely different approach to enhance system capacity and link throughput is to use imaging techniques, which can separate the optical signals impinging from different sources $[14,15]$. The optical signals from two different sources would excite different regions of an imaging concentrator. As such, the data from different streams can be selected or rejected independently. However, such approach would reduce the effective receiver area for the intended signal and therefore require longer integration time for signal detection. Furthermore, the cost of the imaging concentrators would be significantly higher than a standard PD used in optical receivers. Hence, in order to enhance bandwidth reuse and mitigate interference in a cost-effective manner, spectrum sensing approaches would be needed. To this end, the classical carrier sense multiple access (CSMA)/collision detection (CD) approach is considered for optical wireless applications [16]. However, it is well known that the CSMA/CD approach suffers from hidden node and exposed node problems, both of which degrade the performance in an wireless network.

To address the above shortcomings, interference aware allocation of time-frequency slots (chunks) using $\mathrm{BB}$ signalling for transmitting data in optical IM/DDbased OFDMA-time division duplex (TDD) systems is considered in this article. With the proposed approach, the assignment of chunks in a tagged AP is adjusted dynamically depending on the location of an active user in the neighboring cell. To facilitate this, each user equipment (UE) must broadcast a $\mathrm{BB}[17,18]$ in a timemultiplexed slot after successfully receiving data in order to reserve the chunk for the next frame. The AP that intends to transmit on a given chunk must listen to the $\mathrm{BB}$ slot corresponding to that chunk. Provided that TDD channel reciprocity [19] holds, the AP infers the amount of CCI it could potentially cause towards the user that has reserved the chunk. This is vital information that allows an AP to decide without any central supervision whether to transmit or defer the transmission to another time and/or frequency slot so as to limit the CCI caused to the active link to a threshold value. The impact of this threshold parameter on the performance of optical OFDMA networks deployed in a cabin of an aircraft is investigated. Extensive system level simulations demonstrate that performing chunk allocation using the $\mathrm{BB}$ protocol enhances the mean system throughput by $17 \%$, whilst maintaining the same throughput at the cell-edge compared to that achieved with static resource partitioning. In addition, it is found that when the offered load begins to exceed the traffic capacity, hardly any chunks available for a tagged AP are idle. As a result, a new user entering the network or an existing user switching from idle (empty buffer) to active (with a packet in the buffer) state would suffer outage. A heuristic that annuls reservation after a user has had its fair share of resources is proposed. Simulation results show that the BB protocol combined with the proposed heuristic significantly improves both the guaranteed user throughput and the median system throughput compared to the static chunk allocation using cluster-based resource partitioning.

The remainder of the article is structured as followsThe optical wireless network considered in this article is discussed in Section 2. The dynamic chunk allocation (DCA) algorithm using BB signalling and user scheduling is described in Section 3. The simulation parameters and the results are presented in Section 4. Finally, the conclusions are drawn in Section 5. 


\section{System model}

An optical wireless network where $U$ users are served by $N_{\mathrm{A}}$ optical APs is considered for the downlink mode. The transceiver module consists of an array of LEDs and a PD for transmitting and receiving optical signals respectively. An OFDMA-TDD air interface is considered, where the available system bandwidth $B$ is divided into $N_{\mathrm{sc}}$ subcarriers.

The baseband symbols are modulated into an OFDM symbol using inverse discrete Fourier transform (IDFT) and converted into analog signal by digital to analog (D/ A) converter. The LED transmits an optical signal whose intensity is directly proportional to the driving current (i.e. IDFT output). The analog signal transmitted by the LED is given by

$$
x_{t}=\rho \frac{1}{N_{\mathrm{SC}}} \sum_{k=-N_{\mathrm{sc}} / 2}^{N_{\mathrm{sc}} / 2-1} X_{k} e^{-j 2 \pi k \frac{B}{N_{\mathrm{SC}}} t},
$$

where $x_{t}$ is the instantaneous intensity of optical signal emitted by LED at time instant $t ; \rho$ is the scaling factor that maps the IDFT output to analog optical signal; $X_{k}$ is the data symbol transmitted on subcarrier $k$, which is taken from a normalised symbol constellation such that $\operatorname{Var}\left\{X_{k}\right\}=1 \mathrm{~W}$. The total signal power transmitted per subcarrier is $P_{\mathrm{opt}}^{2} / N_{\mathrm{sc}}$, where $P_{\mathrm{opt}}$ is the standard deviation of $x_{t}$. Hence, the scaling factor $\rho$ is set to $P_{\mathrm{opt}}^{2} / N_{\mathrm{sc}}$. In general, the IDFT operation results in complex numbers. However, the optical output is constrained to real and positive numbers. Therefore, $X_{-k}$ is set to $X_{-k}^{*}$ as depicted in Figure $1 \mathrm{a}, \mathrm{X}_{-N \mathrm{sc} / 2}$ is set to 0 and $x_{0}$ is set such that clipping of the OFDM signal is avoided. The above restriction limits the number of chunks that can be independently allocated to half of what is available within the system bandwidth. The transmitted signal $x_{t}$ propagates through the wireless channel and impinges upon the PD at the receiver. The overall channel gain between the LED and the PD is denoted by $G$, which is expressed as [11]

$$
G=\frac{(m+1)}{2 \pi} \cos ^{m}(\phi) g^{\mathrm{pl}} A_{\mathrm{pd}} T_{\psi}^{\mathrm{s}} g_{\psi}^{\mathrm{c}} \cos (\psi),
$$

where $m$ is the mode number of the radiation lobe given by $-\ln 2 / \ln (\operatorname{cod} \Phi)$, where $\Phi$ is the angle at which the radiated power reduces to half compared to the power radiated along the normal of the plane containing the LED; the angles $\varphi$ and $\psi$ are as depicted in Figure 2; $g^{\mathrm{pl}}$ is the optical path loss between the LED and PD which models the propagation of optical signal in space; $A_{\mathrm{pd}}$ is the area of the PD; $T_{\psi}^{s}$ models the signal transmission through the optical filter at the PD and $g_{\psi}^{c}$ is the concentrator gain of a non-imaging concentrator.

The received (optical) signal is converted into an electrical current by a PD whose responsitivity is $R_{\mathrm{pd}}$. The current signal is converted into voltage signal by a transimpedance amplifier (TIA) whose gain is $R_{\mathrm{F}}$. The electrical signal at the TIA output is expressed as

$$
Y_{t}=R_{\mathrm{F}}\left(R_{\mathrm{pd}} G x_{t}+\Omega_{t}\right)
$$

where $\Omega_{t}$ is the additive white Gaussian noise (AWGN) noise which is dominated by shot noise of the PD and the thermal noise of the resistor of the TIA. The received signal is sampled, quantised and passed through fast Fourier transform (FFT) block. Assuming that the $\mathrm{D} / \mathrm{A}$ and analog to digital (A/D) converters are time and frequency synchronised, the output of the discrete Fourier transform (DFT) operation is given by

$$
Y_{k}=R_{\mathrm{F}}\left(R_{\mathrm{pd}} G X_{k}+\Omega_{k}\right)
$$

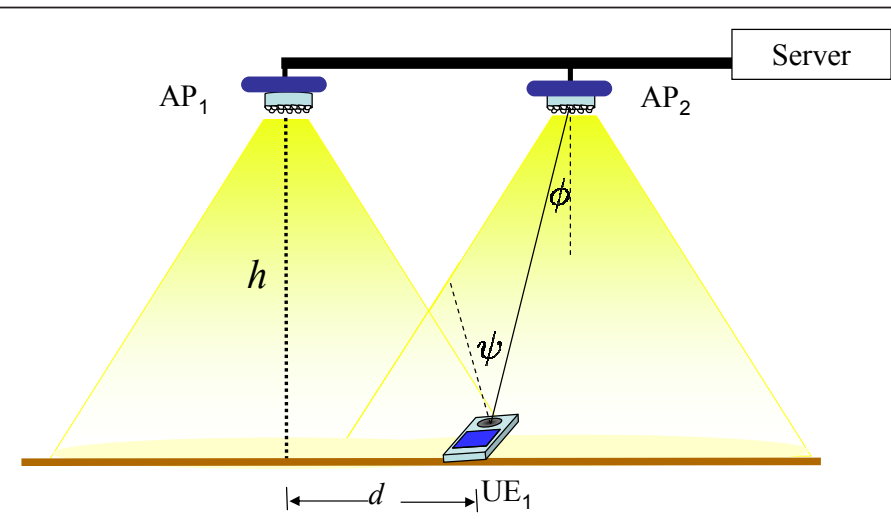

Figure 1 Depiction of physical layer signal processing at the transmitter for data transmission using OFDM modulation in optical wireless system. To maintain real signal, a complex conjugate of baseband symbol applied on subcarrier $k$ must be applied on subcarrier $-k$. Likewise, a DC bias is added to ensure that the signal is positive in order to transmit the signal using IM on subcarrers with index $-N_{s c} / 2$ and 0 . 


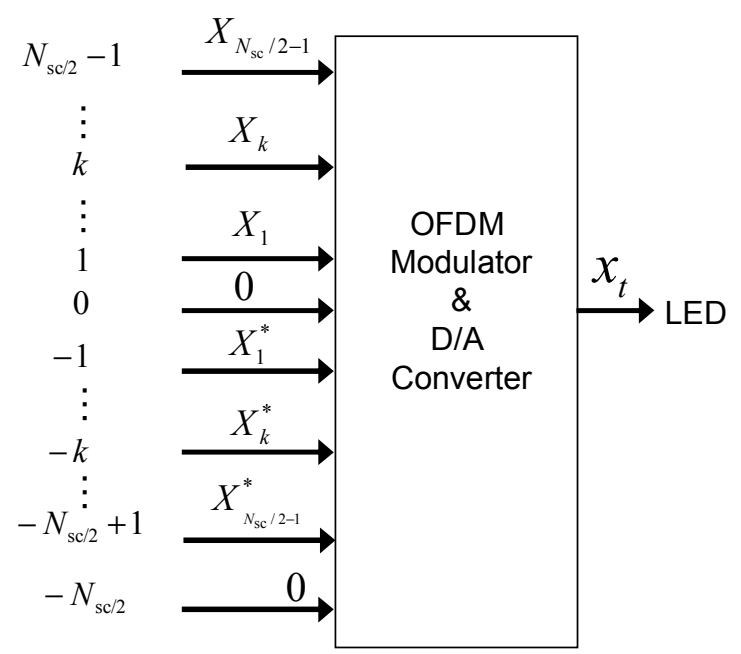

(a) OFDM modulation for optical wireless

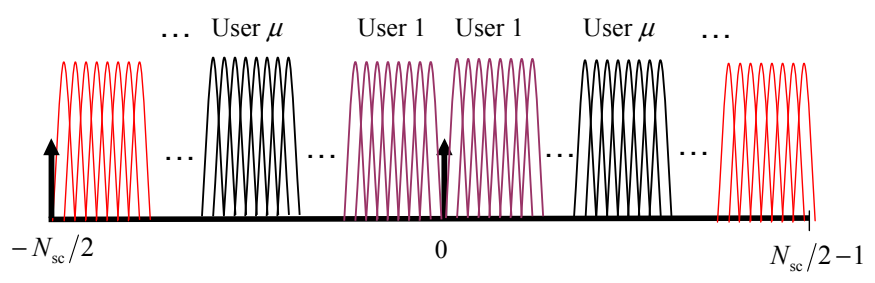

(b) Assignment of subcarriers in optical OFDM

Figure 2 Depiction of interference in optical wireless network for the downlink. $\mathrm{AP}_{1}$ is the intended transmitter for the receiver labeled $U E_{1}$ and $A P_{2}$ is an interfering transmitter, which serves a different $U E$.

where $\Omega_{k}$ is the noise observed on subcarrier $k$, whose power $\mathcal{N}=E\left[\Omega_{k}\right]^{2}$ is expressed as

$$
\mathcal{N}=2 q R_{\mathrm{pd}} P_{\mathrm{am}} B_{\mathrm{sc}}+\frac{4 k_{\mathrm{B}} T B_{\mathrm{sc}}}{R_{\mathrm{F}}}
$$

where $q=1.6 \times 10^{-19} \mathrm{C} ; P_{\mathrm{am}}$ is the intensity of ambient light incident on the PD; $k_{\mathrm{B}}$ is the Boltzmann's constant; $T$ is the absolute temperature and $B s c$ is the bandwidth of a subcarrier. In this article, clipping noise [9] and noise due to the non-linear transfer function of the LED [20] are ignored as these parameters are not central to the contributions of this article.

\section{Interference coordination in optical cells}

A simplified model of the optical wireless cellular system considered in this article is depicted in Figure 2. The available OFDMA subcarriers are grouped in contiguous blocks made up of $n_{\mathrm{sc}}$ subcarriers and $n_{\mathrm{os}}$ OFDM symbols. Such blocks form a resource unit called a chunk and is denoted $(k, n)$ where $k$ is the frequency index and $n$ is the time index. Let $\mu$ denote a UE which is associated with an $\operatorname{AP} \alpha$. Likewise, UE $v$ is another $\mathrm{UE}$ which is served by $\operatorname{AP} \beta$, where $\beta \neq \alpha$ using the same chunk that is used by AP $\alpha$ to serve UE $\mu$. Therefore, AP $\alpha$ causes CCI to UE $v$ and AP $\beta$ causes CCI to UE $\mu$.

In order to distinguish between the intended and the interfering signals, the channel gains (2) are distinguished by adding subscripts of the form $G_{\alpha, \mu}$ where the first subscript denotes the transmitter and the second one denotes the receiver. Likewise, a transmitter index is added to the transmitted symbol to distinguish the symbols transmitted by different transmitters. To this end, $X_{\alpha}$ is used to denote the symbol transmitted by transmitter $\alpha$. The subcarrier indices are omitted for clarity, since the equations apply to an arbitrary chunk in the system. From the perspective of UE $\mu$, the desired and interfering signal power can be expressed as

$$
\begin{aligned}
& R_{\mu}^{\mathrm{d}}=\mathrm{E}\left[\left|Y^{\mathrm{des}}\right|^{2}\right]=\mathrm{E}\left[\left|R_{\mathrm{F}} R_{\mathrm{pd}} G_{\alpha, \mu} X_{\alpha}\right|^{2}\right] \\
& I_{\mu}^{\mathrm{d}}=\mathrm{E}\left[\left|Y^{\inf }\right|^{2}\right]=\mathrm{E}\left[\left|R_{\mathrm{F}} R_{\mathrm{pd}} G_{\beta, \mu} X_{\beta}\right|^{2}\right] .
\end{aligned}
$$

Since the subcarriers are assigned in chunks, the signal-to-interference-plus-noise ratio (SINR) is constant 
for all subcarriers within the chunk. The SINR at UE $\mu$ on chunk $(\kappa, n)$ is expressed as

$$
\begin{aligned}
\gamma_{\mu}[\kappa, n] & =\frac{R_{\mu}^{\mathrm{d}}[\kappa, n]}{I_{\mu}^{\mathrm{d}}[\kappa, n]+\mathcal{N}} \\
& =\frac{\left(R_{\mathrm{pd}} G_{\alpha, \mu}[\kappa, n] \rho\right)^{2}}{\left(R_{\mathrm{pd}} G_{\beta, \mu}[\kappa, n] \rho\right)^{2}+\mathcal{N}}
\end{aligned}
$$

where it is assumed that the transmit power (electrical) on each subcarrier is $\rho^{2}$.

The received signal must be received with a certain minimum SINR that corresponds to the modulation and coding format used for transmission to decode the received signal with a bit error ratio (BER) lower than a tolerated limit. The maximum tolerable BER figure depends on the application and in general it is considered to be $10^{-3}$ for voice and $10^{-7}$ for data. The transmitter and receiver pair can communicate with each other as long as the minimum SINR target needed for decoding the lowest order modulation and coding scheme with the required quality of service (QoS) is met. To enable the SINR target to be met, the allocation of resources among the links closely located in space needs to be coordinated. One means to coordinate the resources in a self-organising and decentralised manner is to apply the $\mathrm{BB}$ protocol for interference aware resource allocation in the system.

Interference coordination using BB signalling comprises of the following key components, namely

(1) Interference avoidance: The transmitter avoids causing detrimental CCI towards the receivers of preestablished links. This is achieved by sensing the BB signal to determine the idle chunks as described in Section 3.1.

(2) Contention mitigation: The access of chunks that are not yet reserved by transmitting a BB signal is regulated such that at most one transmitter within a coordination cluster may access the idle chunks. Such coordination is required so as to ensure that no two transmitter sense the same chunk as idle and cause collision of data in the next frame. This procedure is described in Section 3.2.

(3) Interference aware scheduling: The transmitter does not schedule transmissions for a user on the chunks where the receiver senses high level of CCI originating from pre-established links, even if the transmitter has sensed the chunk to be idle. In a multi-user cellular system, such as the one considered in this article, the chunk can be reused at the tagged AP by allocating it to a different user that reports a lower level of $a$ priori CCI. This procedure is described in Section 3.4 .

\subsection{Interference coordination using BB signalling}

For interference coordination among optical wireless cells, the medium access control (MAC) frame is divided into data and BB slots. Data transmission is carried out during the data slot and the $\mathrm{BB}$ is transmitted during the $\mathrm{BB}$ mini slot. Each receiver must transmit a $\mathrm{BB}$ to announce its presence to other APs so that they may autonomously determine whether or not they can reuse the reserved chunk. To this end, it is assumed that UE $\mu$ has transmitted a BB after receiving data during its data slot. The busy burst signal transmitted by UE $\mu$ propagates through the optical channel and is received both at APs $\alpha$ and $\beta$. The received signal at the serving AP $\alpha$ informs the transmitter that the minimum SINR target has been met. Furthermore, additional information can be piggybacked to the BB signal for feedback and control purposes. By contrast, the received BB signal at AP $\beta$ makes it aware of the amount of CCI it potentially causes to the active receiver $\mu$ if it were to reuse the reserved resource. By measuring the signal power received during the $\mathrm{BB}$ slot, the AP $\beta$ decides autonomously whether or not it may reuse the reserved chunk so as to limit the interference to a threshold value. To this end, the received $\mathrm{BB}$ signal at AP $\beta$ can be written as

$$
y_{k}^{\mathrm{bb}}=R_{\mathrm{F}} R_{\mathrm{pd}} G_{\mu, \beta} x_{k, \beta}^{\mathrm{bb}} .
$$

The power of the $\mathrm{BB}$ signal is given by

$$
I_{\beta}^{\mathrm{bb}}=\mathrm{E}\left[\left|\gamma_{k}^{\mathrm{bb}}\right|^{2}\right]=\left(R_{\mathrm{F}} R_{\mathrm{pd}} G_{\mu, \beta} \rho^{\mathrm{bb}}\right)^{2} .
$$

Combining (7) and (10) and provided that $G_{\beta, \mu}=G_{\mu, \beta}, I_{\mu}^{\mathrm{d}}$ can be expressed in terms of $I_{\beta}^{\mathrm{bb}}$ as

$$
I_{\mu}^{\mathrm{d}}=\left(\frac{\rho}{\rho^{\mathrm{bb}}}\right)^{2} I_{\beta}^{\mathrm{bb}} .
$$

The condition $G_{\beta, \mu}=G_{\mu, \beta}$ holds true if either $m=1$, which is the case for Lambertian transmitters $\left(\varphi=60^{\circ}\right)$ or if $\varphi=\psi$, which is true when the transmitter plane and the receiver plane are parallel to each other.

$$
\begin{aligned}
\text { If } \rho & =\rho^{\mathrm{bb}}, \text { from (10) and (11), } \\
I_{\mu}^{\mathrm{d}} & =I_{\beta}^{\mathrm{bb}} .
\end{aligned}
$$

To ensure that $I_{\mu}^{\mathrm{d}} \leq I_{\text {th }}$ AP $\beta$ may reuse the resource if $I_{\mu}^{\mathrm{d}} \leq I_{\text {th }}$. Furthermore, provided that both the transmit data power and the BB power (in electrical domain) are equal, the condition for reusing a resource is given by

$$
I_{\beta}^{\mathrm{bb}} \leq I_{\mathrm{th}} .
$$


Hence, by measuring the $\mathrm{BB}$ power observed during the feedback slot, AP $\beta$ determines whether or not it causes detrimental CCI (i.e. CCI higher than the predetermined threshold value) towards the active user $\mu$ served in an adjacent cell $\alpha$. For a given threshold $I_{\text {th }}$, (13) is more likely to hold true if $\mu$ lies close to AP $\alpha$ compared to the case if $\mu$ lies at the boundary of the coverage regions of APs $\alpha$ and $\beta$. Therefore, each AP can dynamically determine the set of chunks it can use for transmission whilst avoiding detrimental CCI to preestablished links simply by measuring the received $\mathrm{BB}$ power and comparing it against the threshold value and without requiring a central coordinator.

The $\mathrm{BB}$ protocol relies on the assumption that AP $\alpha$ and AP $\beta$ do not check (13) simultaneously. If both AP $\alpha$ and AP $\beta$ were to sense the channel simultaneously, they would both infer that the channel is free, since there is no pre-established receiver in the vicinity that announces its presence by emitting a BB signal. In such scenario, they would both schedule transmission for their own users without any knowledge of the user the other AP might schedule. Hence, each of the APs can potentially cause severe CCI to the user served by the other AP. Such collision can potentially cause high outage particularly at the cell-edge. To mitigate such problem, the cellular slot access and reservation (CESAR) approach [21] is used, which ensures that an idle chunk is not assigned simultaneously by neighbouring APs through a cyclic shifted allocation pattern for idle chunks.

\subsection{Contention avoidance among neighbouring cells}

The contention problem can be mitigated by exploiting the properties of a cellular network using CESAR approach proposed in our earlier study [21]. In summary, the available chunks within the system bandwidth are grouped into $R$ different subbands. Likewise, the cells in the system are also grouped into $R$ different groups $\mathcal{G}$, such that the APs within a coordination cluster belong to different groups. For the optical network, a reasonable choice of the group size is $R=3$. At time instant $n$, the APs $\beta$ that belong to group $g_{\beta}[\kappa, n] \in \mathcal{G}$ may access idle chunk $(\kappa, n)$ in a predefined cyclic manner, determined by [21]

$$
g_{\beta}[\kappa, n]=\bmod (n+\kappa, R)
$$

The term $g_{\beta}[\kappa, n] \in \mathcal{G}$ determines whether or not a chunk $(\kappa, n)$ may be accessed by AP $\beta$. Hence, (14) establishes a schedule to access idle chunks, i.e. chunks that are not protected by transmitting the BB. Thus, the CESAR mechanism ensures that at a particular time instant $k$ only one group of APs may access idle resources, thereby mitigating the contention problem.
Cyclicly shifting the AP groups in (14) over time ensures that after $R$ slots an AP is granted access to all chunk that are sensed idle. The APs are numbered such that an AP is associated to its group by the relation $\mathcal{G}=\{\bmod (\beta, R)\}$. Then, the AP $\beta$ may allocate chunk $(\kappa, n)$ to user $v$ only if both of the following conditions hold:

(1) $\bmod (\beta, R)=\bmod (n+k, R)$ indicates that AP $\beta$ may access chunk $(\kappa, n)$.

(2) The threshold test (13) applied on chunk $(\kappa, n)$ holds true.

The second condition indicates that chunks already reserved by transmission of a BB signal retain unrestricted access to a given frequency resource unit $k$, and continue to serve the users that have reserved those chunks. CESAR [21] and BB protocol for interference coordination in optical wireless network perfectly complement each other; the former mitigates collisions due to simultaneous access in contention through (14), while the latter facilitates interference aware selection of chunks reserved by transmitting the BB signal.

\subsection{Avoiding $\mathrm{CCl}$ from preestablished links}

The mechanism discussed earlier focussed on how an AP can determine the set of chunks that it is permitted to use so as to limit the CCI caused to users served by the neighbouring APs to a threshold value. However, UE $v$ also suffers from CCI originating from pre-established links, which make no effort to limit the CCI caused to the newly entering link. Hence, the minimum SINR target may not be met for the cell-edge user served by the tagged AP if the user is served using the chunks that are in use in the neighbouring cell. To avoid scheduling UE $v$ on a chunk where the CCI caused by pre-established communication links would cause the minimum SINR target not to be met, an $a$ priori estimate of SINR $\hat{\gamma}_{\nu}[\kappa, n]$ is made as follows

$$
\hat{\gamma}_{\nu}[\kappa, n]=\frac{R_{\nu}^{\mathrm{d}}[\kappa, n]}{I_{\nu}^{\mathrm{d}}[\kappa, n-1]+\mathcal{N}^{\prime}}
$$

where $I_{v}^{\mathrm{d}}[\kappa, n-1]$ is the interference observed on the chunk $(\kappa, n-1)$. To ensure that the collisions are mitigated, the chunk can be assigned to user $v$ only if

$$
\hat{\gamma}_{\nu}[\kappa, n] \geq \Gamma_{\min }
$$

This information can be transmitted to the serving AP either using piggyback signalling or via dedicated control channel.

\subsection{User scheduling}

The score-based scheduler with reservation [18] is used for distributing $N_{\mathrm{C}}$ chunks among $U_{\mathrm{S}}$ users served by an 
AP. The users compete for being scheduled on chunks that are not yet reserved for any user served by a given AP. Numerical scores are calculated for each user on each chunk which serves as a quantitative basis for ranking each user's suitability for being scheduled on each chunk. The user that is scheduled on chunk $(\kappa, n)$ is defined as

$$
\zeta_{\beta}[\kappa, n]= \begin{cases}\underset{v}{\arg \min } s_{v, \beta}[\kappa, n], & b_{v}[\kappa, n-1]=0 \quad \forall v \in\left\{1 \ldots U_{s}\right\} \\ v_{v} & b_{v}[\kappa, n-1]=1,\end{cases}
$$

The reservation indicator on chunk $(\kappa, n), b_{v}[\kappa, n]$, is set depending on the SINR achieved at the receiver during the data transmission slot, as follows

$$
b_{v}[\kappa, n]=\left\{\begin{array}{l}
1, \gamma_{\nu}[\kappa, n] \geq \Gamma_{\min } \text { and } \zeta_{\beta}[\kappa, n]=v \\
0, \text { otherwise }
\end{array}\right.
$$

where $b_{v}[\kappa, n]=1$ indicates that the user $v$ has reserved the chunk $(\kappa, n+1)$ by transmission of $\mathrm{BB}$ during the BB mini slot associated to chunk $(\kappa, n)$. For these reserved chunks, the modulation format is updated using the link adaptation algorithm proposed in Section 3.5. All users served by the tagged AP compete for getting access to the idle chunks in the system. The score for each user $s_{v, \beta}$ on each chunk is calculated by taking instantaneous fairness in the system and by respecting any restriction imposed on accessing the chunk. The score to be used in (17) for user $v$ served by AP $\beta$ for chunk $(\kappa, n)$ is calculated as

$$
s_{v, \beta}[\kappa, n]=\epsilon_{v, \beta}[\kappa, n]+\Psi_{\nu}
$$

where the term $\varepsilon_{v, \beta} \in\{0, \infty\}$ indicates whether an idle chunk $(\kappa, n)$ (i.e. $b_{v}[\kappa, n-1]=0$ in (17)) may be accessed by user $v$, given by

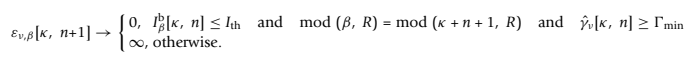

In (20), the first relation evaluates the threshold test (13) for interference aware beam selection; the second relation determines if the AP $\beta$ is granted access to idle chunk $(\kappa, n+1)$, according to the CESAR principle described in Section 3.2; whereas the third relation ascertains that the estimated SINR at the receiver exceeds the minimum SINR target to avoid interference from preestablished links to the tagged link. If $\varepsilon_{v, \beta}[\kappa, n$ $+1] \rightarrow \infty$ is set for all $U_{\mathrm{S}}$ users, the chunk remains idle in sector $\beta$, so that $\zeta_{\beta}[\kappa, n+1]=\varnothing$ in (17).

Likewise, the term $\Psi_{v}$ is a priority penalty factor for user $v$. Each user is assigned the chunk which the aforesaid user had reserved by transmitting a BB. The penalty factor ensures that the users that have fewer chunks already reserved have higher priority in accessing idle chunks. To this end, $\Psi_{v}$ is initialized at the start of the scheduling process, as follows

$$
\Psi_{v}=\exp \left(\sum_{\kappa=1}^{N_{C}} b_{\nu}[\kappa, n]\right) .
$$

When an idle chunk is assigned to user $v$ by (17), $\Psi_{v}$ is updated, as follows

$$
\Psi_{\nu} \leftarrow \Psi_{\nu} \exp (1),
$$

so that the users that have fewer chunks already assigned are preferred to be scheduled on subsequent slots.

In the scheduler considered above, the chunks are reserved by a user by transmitting a $\mathrm{BB}$ as long as it has data to transmit. If the user that has successfully accessed the chunk has no more data to transmit, the reservation is annulled and the chunk may be reassigned to another user. Unfortunately, the scheduler does not preempt any user with heavy traffic volume from reserving all the chunks within the system bandwidth. Thus, such users will cause a new user entering the network or a user switching from idle state (empty transmit buffer) to active state (at least a packet in the transmit buffer) to find that all the chunks in the network are busy, thereby causing high outage. To address such problem, a fair reservation mechanism is proposed where the reservation made by the user by transmitting a BB is annulled by the serving AP immediately after finding that the number of slots a chunk has been reserved by a user exceeds the reservation threshold. This is achieved by setting the reservation indicator $b_{v}[\kappa, n]$ as follows

$$
b_{v}[\kappa, n]=\left\{\begin{array}{l}
1, \gamma_{v}[\kappa, n] \geq \Gamma_{\min } \text { and } \zeta_{\beta}[\kappa, n]=v \text { and }\left(\sum_{\ell=1}^{R_{\mathrm{h}}} \mathrm{b}_{v}[\kappa, \ell]\right)<R_{\mathrm{th}}, \\
0, \text { otherwise }
\end{array}\right.
$$

where $R_{\mathrm{th}}$ is the reservation threshold which is a user specific parameter. It is proposed that the $R_{\text {th }}$ parameter should be set to

$$
R_{\mathrm{th}}=\left\lfloor\frac{N_{\mathrm{C}}}{\# \mathcal{A}_{v}[n]}\right\rfloor,
$$

where $\lfloor()$.$\rfloor is the floor operator, \mathcal{A}_{v}[n]$ is the set of chunks assigned to user $v$ at time instant $n$ and $\# \mathcal{A}_{v}$ expresses the number of chunks assigned to user $v$.

\subsection{Link adaptation}

Let $\mathcal{M}=\{1, \ldots, M\}$ be the set of supported modulation schemes. Associated to each modulation scheme $m \in M$ is an SINR target $\Gamma=\left\{\Gamma_{1}, \ldots, \Gamma_{M}\right\}$ that must be achieved to satisfy a given frame error rate (FER). The objective is to select the modulation scheme $m_{\nu}[\kappa, n] \in \mathcal{M}$ for chunk $(\kappa, n)$, which yields the highest spectral efficiency, for which $\gamma_{\nu}[\kappa, n] \geq \Gamma_{m_{\nu}[\kappa, n]}$ 
holds. Assuming that the channel does not change significantly between two consecutive time frames, feedback of SINR observed in the preceding frame is used to select appropriate modulation format for the next frame. It is sufficient to provide quantised feedback using $\left\lceil\log _{2}(M)\right\rceil$ bits, where $\lceil()$.$\rceil is the ceiling operator.$ Such quantised feedback may be piggybacked to the BB signal. Therefore, additional overhead involved in dedicating one OFDM symbol for accommodating the $\mathrm{BB}$ mini-slot is compensated by utilising the BB for signalling purposes. In order to determine a suitable modulation format, the following steps are carried out at the UE (except Step 3 which is carried out at the $\mathrm{AP})$ :

(1) Conduct an a priori estimate of the SINR on chunk $(\kappa, n)$ based on the interference measured in the preceding frame

(2) Determine the largest order modulation scheme, $\hat{m}$, which fulfils $\hat{\gamma}_{\nu}[\kappa, n] \geq \Gamma_{\hat{m}[\kappa, n]}$ using a look up table and feed back $\hat{m}$ to the AP.

(3) Transmit data using $m_{\nu}[\kappa, n]=\hat{m}$ to the intended UE.

(4) Make an a posteriori estimate of the achieved SINR $\gamma_{\nu}[\kappa, n]$ using (9).

(5) Recalculate $\hat{m}$ using the look up table, such that $\gamma_{v}[\kappa, n] \geq \Gamma_{\hat{m}}[\kappa, n]$ holds.

(6) Compute the modulation scheme to be used in the next frame as follows

$$
m_{\nu}[\kappa, n+1]= \begin{cases}\bar{m}, & \gamma_{\nu}[\kappa, n] \geq \Gamma_{m_{v}[\kappa, n]+1} \\ 0, & \gamma_{\nu}<\Gamma_{\min } \\ m_{v}[\kappa, n], & \text { otherwise, }\end{cases}
$$

Where $\left.\bar{m}=\left\lceil m_{\nu}[\kappa, n]+\hat{m}[\kappa, n]\right) / 2\right\rceil$.

(7) If $m_{v}[\kappa, n+1]=0$, or the chunk is no longer needed, do not transmit $\mathrm{BB}$ and release chunk $(\kappa, n)$. Otherwise, transmit BB with $m_{v}[\kappa, n+1]$ piggybacked and go to Step 3.

\subsection{Benchmark system}

Chunk allocation using static resource partitioning using the cluster-based approach $[10,11]$ is considered as the benchmark. $K$ adjacent optical APs form a coordination cluster as depicted in Figure 3a and the available bandwidth is divided equally among the $K$ APs within the cooperation cluster as depicted in Figure 3b. In this article, a cluster size $K=3$ is chosen.

In addition, comparisons are also made against a full chunk reuse system where each AP transmits on the entire available bandwidth without interference coordination among neighbouring cells.

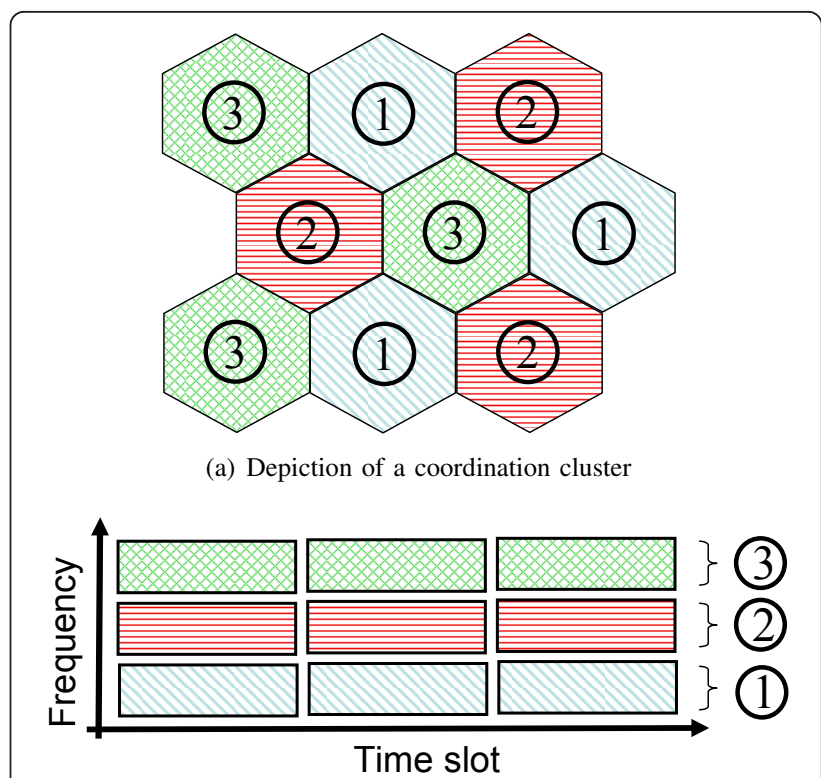

(b) Resource partitioning approach
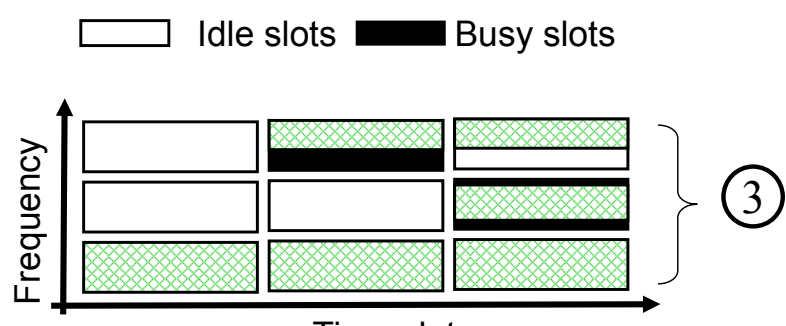

Time slot
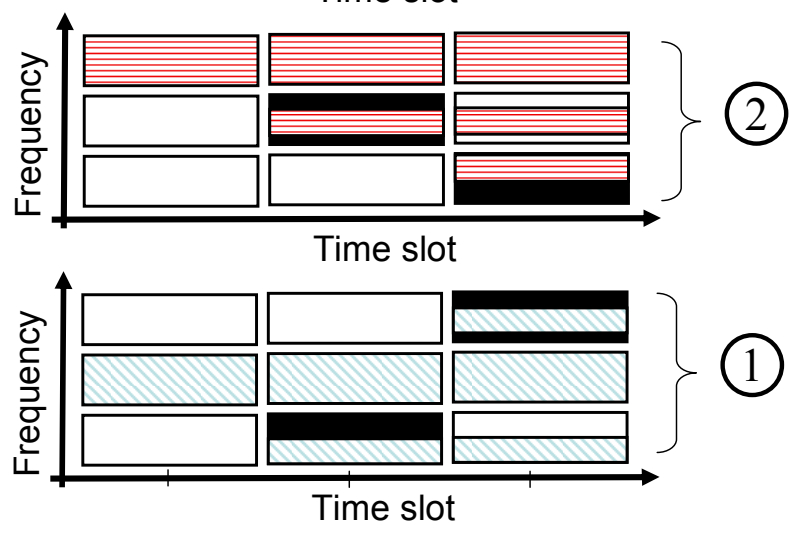

(c) Illustration of CESAR approach

Figure 3 Collision avoidance using cellular slot access and reservation (CESAR) approach [21]. The size of the coordination cluster (a) is set such that any two transmitter and receiver pair that could cause collisions are within the coordination cluster, which is a principle followed for resource partitioning (b). Only one cell within a coordination cluster accesses idle chunks and avoids transmitting on those chunk where BB is sensed above the threshold value. The idle chunks that are successfully accessed are reserved and used for transmission in subsequent slots (c). 


\section{Results and discussions}

The performance of the chunk allocation mechanism using $\mathrm{BB}$ signalling is compared against the bench-mark systems discussed earlier in Section 3.6. The performance metrics considered are user throughput and system throughput, which is an aggregate throughput of all users served by a given AP.

\subsection{Simulation setup}

An optical wireless network deployed inside an aircraft cabin is considered where the APs (denoted by ' + ') are distributed as depicted in Figure 4. The transmitter is an array of LEDs [22] and the receiver contains a single photodiode [23], both of which have peak spectral response at $850 \mathrm{~nm}$. The components for transmitter and receiver are assumed to be identical both at the APs and the UEs.

Users are uniformly distributed in a plane surface at a height of $1 \mathrm{~m}$ from the plane containing the APs. The channels are modelled using the pathloss model reported in [11]. The channels are assumed to be frequency flat and time invariant, which is a reasonable assumption because multipath components cannot be resolved due to the size of the detector area relative to the carrier wavelength. A full buffer traffic model is considered and perfect synchronisation in time and frequency is assumed. Link adaptation is performed using the algorithm proposed in [24] where the available modulation and coding format is matched according to the prevalent channel conditions at the receiver. The simulation parameters are presented in Table 1.

\subsection{Distribution of SINR in space}

The spatial distribution of SINR around each AP is depicted for the downlink (DL) mode in Figure 4. The results show that the SINRs fall as low as $-3.2 \mathrm{~dB}$ on average, given that all the available chunks are reused in each cell. However, when the allocation of chunks is coordinated using a static cluster-based resource partitioning approach, the SINR at the aforesaid location improve to roughly $8 \mathrm{~dB}$. Clearly, the system is limited by interference rather than by noise for the set of system parameters considered. Therefore, the goal is to balance the reuse of chunks with the SINR achieved at the receiver,

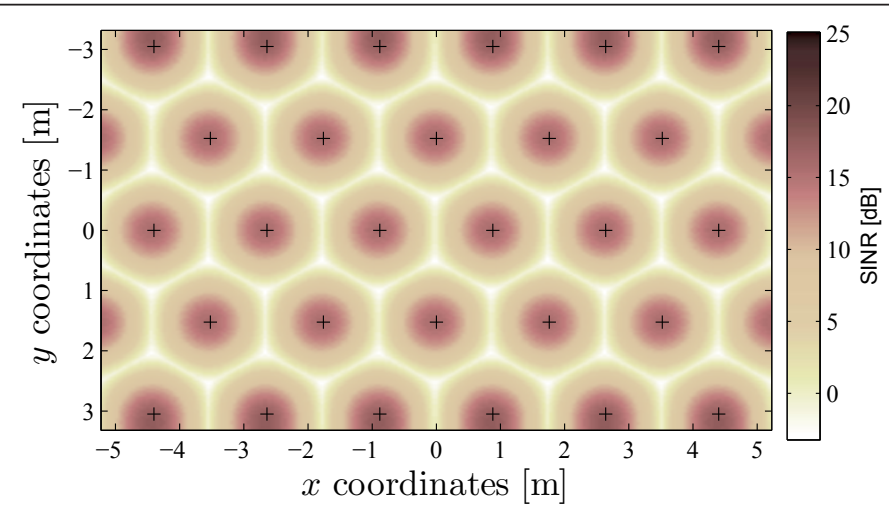

(a) Full reuse $(K=1)$

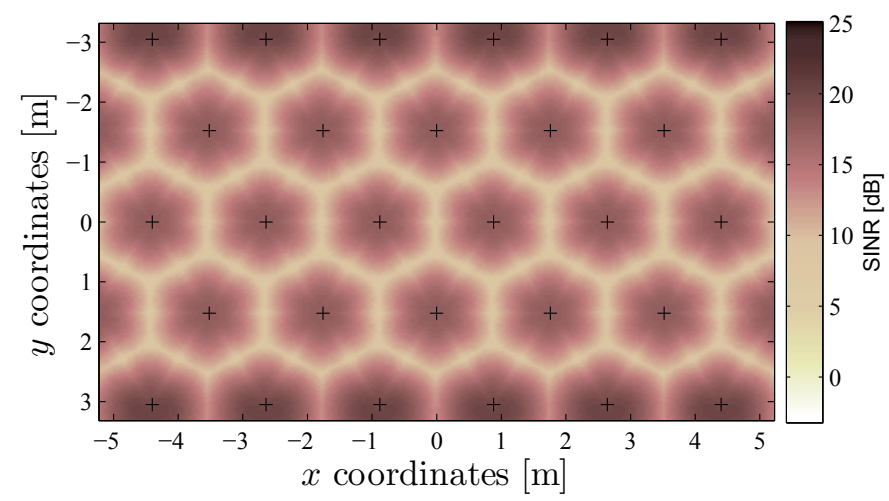

(b) Reuse partitioning $(K=3)$

Figure 4 Map of SINR achieved in an optical wireless network in the horizontal cross section of an aircraft cabin at a height of $1 \mathrm{~m}$ from the transmitter 
Table 1 Simulation parameters

\begin{tabular}{ll}
\hline Parameter & Value \\
\hline Half power beamwidth of LED & $60^{\circ}$ \\
Transmit power (optical) $(P)$ & $1 \mathrm{~W}$ \\
Responsitivity of PD $\left(R_{\mathrm{pd}}\right)$ & $0.63[\mathrm{~A} / \mathrm{W}]$ \\
Area of PD $\left(A_{\mathrm{pd}}\right)$ & $150 \mathrm{~mm}^{2}$ \\
Reflection coefficient of glass $\left(n_{\mathrm{g}}\right)$ & 1.5 \\
Feedback resistance of TIA $\left(R_{\mathrm{F}}\right)$ & $6 \mathrm{k} \Omega$ \\
System bandwidth $(B)$ & $20 \mathrm{MHz}$ \\
Temperature $(T)$ & $300 \mathrm{~K}$ \\
Background radiation $\left(P_{\mathrm{bg}}\right)$ & $6 \mathrm{~mW} / \mathrm{cm}{ }^{2} / \mathrm{nm}$ \\
Bandwidth of optical filter $\left(\lambda_{\text {of }}\right)$ & $300 \mathrm{~nm}$ \\
Coding & $3 / 4-\mathrm{rate}$ convolutional \\
& BPSK, QPSK, cross 8-QAM, \\
Modulation format & $16-\mathrm{QAM}$, cross 32-QAM, 64-QAM, \\
& cross 128-QAM and 256-QAM \\
SINR target $(\Gamma)[\mathrm{dB}]$ & $2.2,5.2,9.1,11.3,14.4,16.6$, \\
& $19.5,22.5$ \\
\hline
\end{tabular}

such that a desirable compromise can be made between the conflicting goals of improving the system throughput and enhancing user throughput at the cell-edge.

\subsection{Performance of $B B$ signalling}

In the following, the performance of $\mathrm{BB}$ signalling is compared against a cluster-based resource partitioning approach as well as against a full chunk reuse approach. For the results presented in this section, the chunk reservation policy is that a user that has transmitted a $\mathrm{BB}$ is assigned a chunk as long as it has additional data to transmit in its buffer.

\subsubsection{Full buffer traffic model}

The performance of the $\mathrm{BB}$ protocol is compared against the benchmark system in Figure 5. The impact of the threshold parameter on the system performance is investigated using four representative values of the $\mathrm{BB}$ specific threshold parameter, namely $-18,-15,-12$, and $-9 \mathrm{dBm}$. The results show that setting the threshold to $-18 \mathrm{dBm}$ enforces the largest exclusion region around the active receiver and therefore leads to highest guaranteed user throughput (measured at $10^{\text {th }}$ percentile of user throughput) among the considered thresholds and the benchmark systems. By setting the threshold to -18 $\mathrm{dBm}$, a guaranteed user throughput of $1.97 \mathrm{Mbps}$ is achieved (see Figure 5b). For comparison, the static frequency planning using a reuse factor 3 also achieves roughly the same cell-edge user throughput. This observation demonstrates that a reuse factor of 3 is optimal for the considered system at the cell-edge since the throughput cannot be improved further by reducing or increasing the reuse of the chunks. Additionally, the interference awareness property of $\mathrm{BB}$ signalling mechanism helps the tagged AP identify the chunks that are allocated to the cell-center users in the neighboring cells and reuses such chunks to serve its own user. Therefore, the $\mathrm{BB}$ protocol is able to improve the throughput of cell-center users (compared to the resource partitioning approach using reuse factor of 3 ) without compromising the cell-edge user throughput. As a result, the system throughput (see Figure 5a) improves by $17 \%$ using $\mathrm{BB}$ protocol compared to using cluster-based resource partitioning approach with a cluster size of 3.

When the threshold is gradually increased, the system throughput increases due to an increase in the spatial reuse of chunks but the throughput at the cell-edge decreases. Initially, the increase in CCI reduces the achieved SINR at the receiver and forces the transmitters to utilise lower order modulation and coding format. However, on further increasing the threshold, the cell-edge users are forced to release the reserved chunks since the minimum SINR target is no longer met. The scheduler then assigns the released chunks to cell-center users where the minimum SINR target is more likely to be met. This is because the CCI caused to the receiver from pre-established transmission in neighboring cell causes the minimum SINR target (16) not to be met for the cell-edge users. As a result, the number of chunks allocated to users close to the serving AP increases at whereas the number of chunks available to the cell-edge users reduces. Hence, the system throughput increases (see Figure 5a) at the cost of cell-edge user throughput (see Figure $5 \mathrm{~b}$ ). In particular, when the interference protection is annulled by setting high thresholds, such as -9 $\mathrm{dBm}$, the performance at the cell-edge approaches that of a full reuse system (see Figure 5b). The released chunks are reallocated to cell-center users, which improves the system throughput at the cost of outage at the cell-edge. The results in Figure 5 depict the various degrees of trade off between interference protection and spatial reuse simply by adjusting the threshold parameter.

\subsubsection{Bursty traffic model}

The results in Section 4.3.1 were obtained assuming a full buffer traffic, which represents an upper limit on the offered load where each user has enough data to fill the entire bandwidth in every slot. For practical application such as accessing multimedia contents, user traffic is composed of Internet protocol (IP) packets. An interesting question would be to quantify the performance of the proposed approach when the traffic is bursty. To investigate the performance, the system is simulated for various offered loads ranging from roughly $10 \mathrm{Mbps} /$ cell to $160 \mathrm{Mbps} / \mathrm{cell}$ and the results are presented in Figure 6. The offered loads are varied by adjusting the interarrival time, which follows an exponential distribution. The results show that 


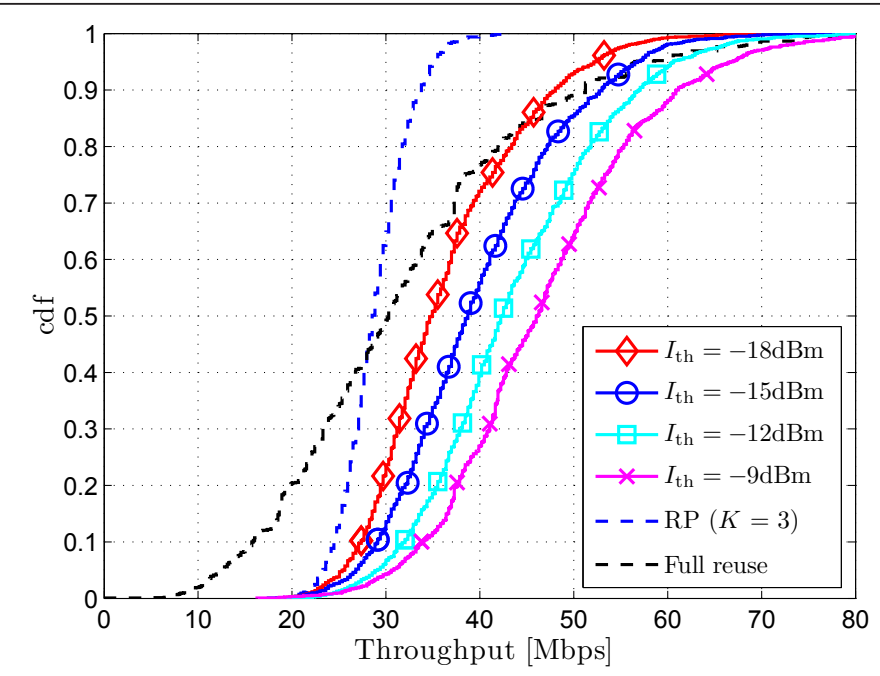

(a) System throughput

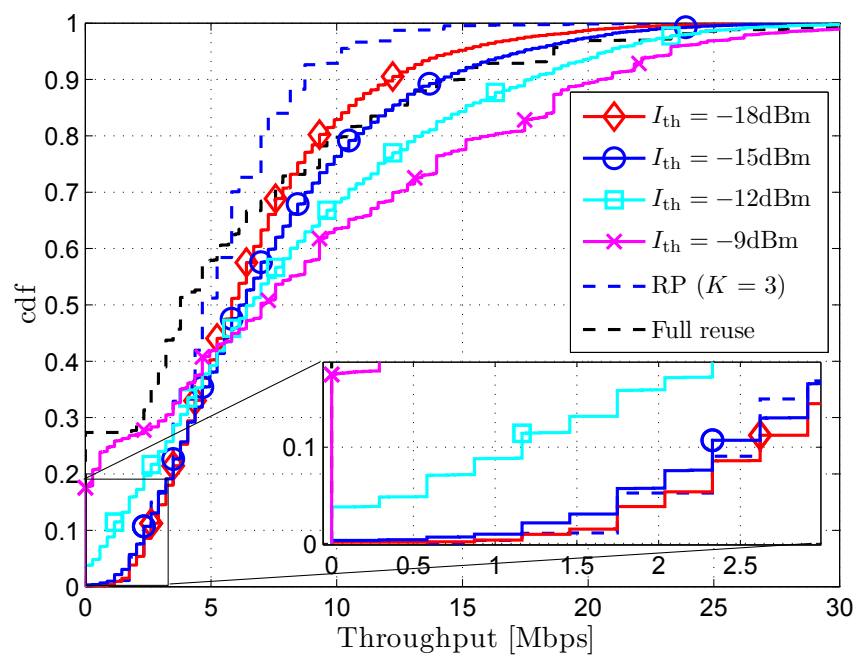

(b) User throughput

Figure 5 Comparison of performance of DCA using BB signalling in optical wireless network. The BB signal correspond to the signal power in the electrical domain after the TIA whose gain is $6 \mathrm{kV} / \mathrm{A}$.

interference coordination improves the performance of the system compared to that achieved without interference coordination (i.e. full chunk reuse). Moreover, it can also be observed that dynamic chunk allocation using the $\mathrm{BB}$ protocol achieves better performance than that is achieved with the cluster-based static chunk allocation using a cluster size of 3 in terms of median system throughput, median user throughput and peak user throughput (See Figure 6b-d). This is attributed to the interference awareness property of the $\mathrm{BB}$ protocol that allows the system to identify the chunks that can be reused in the tagged cell without causing detrimental CCI to the users served by the neighbouring APs. However, an interesting behaviour can be observed with the guaranteed user throughput, i.e. the $10^{\text {th }}$ percentile (Figure $6 \mathrm{a}$ ). When the offered system load is less than $25 \mathrm{Mbps} /$ cell, the chunks in the system are not fully reused and therefore chunk allocation using $\mathrm{BB}$ approach provides the same throughput as the static chunk allocation approach. When the offered load is increased from $25 \mathrm{Mbps} / \mathrm{cell}$ to $40 \mathrm{Mbps} / \mathrm{cell}$, dynamic chunk allocation using the BB protocol delivers a superior performance in terms of peak user throughput, median system throughput and median user throughput (see Figure 6b-d), whilst achieving the same guaranteed user throughput (see Figure 6a). Furthermore, the interference threshold can be adjusted dynamically to tradeoff between the aggregrate system throughput and throughput guaranteed at the cell-edge. 


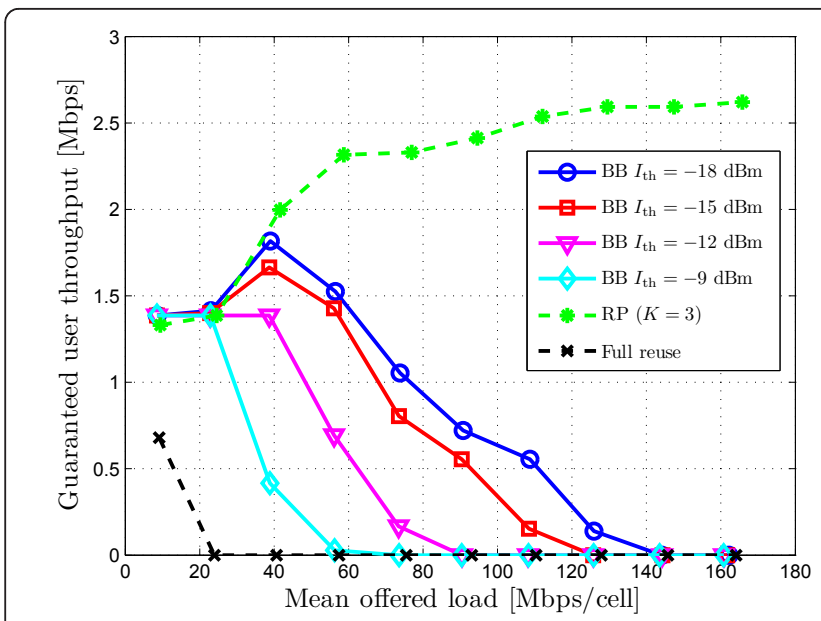

(a) Guaranteed user throughput

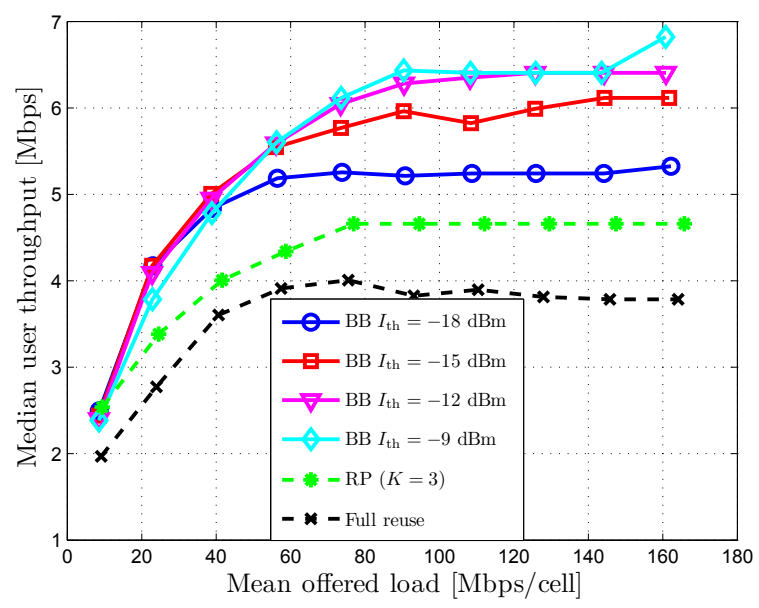

(c) Median user throughput

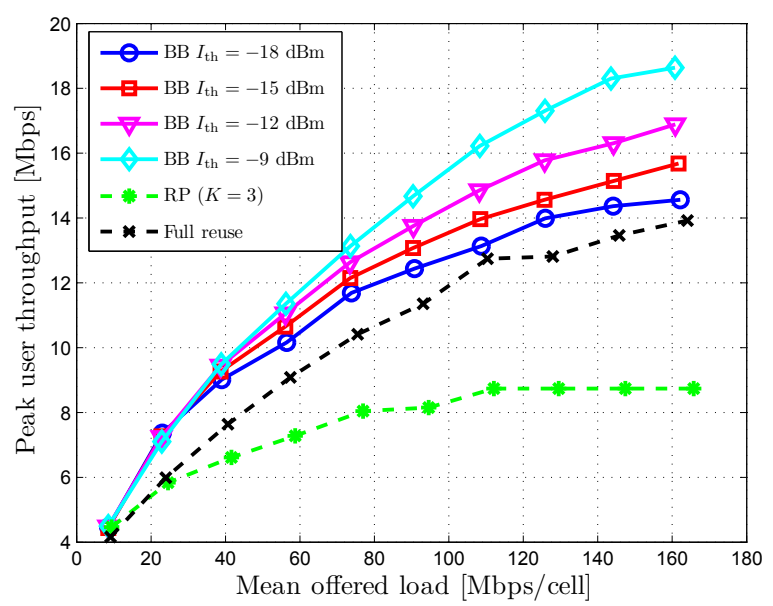

(b) Peak user throughput

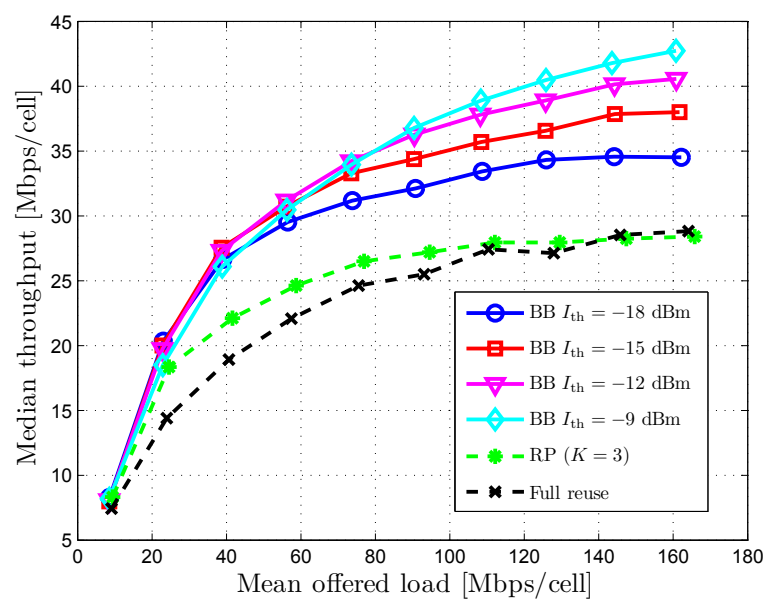

(d) Median cell throughput

Figure 6 Comparison of the performance of chunk allocation using BB signalling where the successfully accessed chunks are reserved until the transmit buffer is empty for various traffic loads against the benchmark systems.

However, when offered load is higher than $40 \mathrm{Mbps}$, the guaranteed user throughput using the BB protocol degrades with an increase in the offered load. By contrast, the guaranteed user throughput with static chunk allocation increases until a peak is reached and roughly the same level of throughput is maintained. Note that the system bandwidth is only $20 \mathrm{MHz}$, out of which only $10 \mathrm{MHz}$ can be allocated independently. The remaining $10 \mathrm{MHz}$ of the system bandwidth is used to transmit the complex conjugate of data symbols in order to maintain a real valued signal. With these parameters, the peak raw data rate in an isolated cell would be 10 Mbps assuming a binary phase shift keying (BPSK) modulation and $80 \mathrm{Mbps}$ assuming 256-quadrature amplitude modulation (QAM) modulation format. Therefore, this reflects a scenario where a user with heavy traffic demand competes with other users that may possibly have heavy or light traffic demands. Provided that the user with heavy traffic demand has successfully accessed the chunk and reserved it by transmitting a $B B$ signal, the chunks available at the AP are exclusively assigned to that user. Such chunks appear unavailable to the user that has just entered the network or switched from idle (empty transmit buffer) to active (containing at least a protocol data unit (PDU) queued in the transmit buffer). Likewise, assuming that an active user releases a chunk when its transmit buffer is empty, it will find that the chunks are all occupied at a later point in time when such user attempts to transmit data again. Moreover, increasing the traffic load increases the number of frames that a chunk reserved by a user appears unavailable to other users. Thus, the ability of a user to reacquire the released chunks decrease with 
an increase in traffic load. Therefore, the guaranteed user throughput decreases.

The assumption that the reserved chunk will be allocated to the same user in the next slot ensures that the a priori knowledge of the amount of CCI caused to the user served by a neighbouring cell is valid. However, the results presented in Figure 6 have demonstrated that allowing for the reservation of chunks until the transmit buffer is emptied deteriorates the system performance once the system is overloaded. To address this shortcoming, a fair chunk reservation mechanism was proposed in Section 3.4, whose performance is analysed in the next section.

\subsection{Performance of $B B$ signalling with fair reservation mechanism}

The performance of $\mathrm{BB}$ protocol with fair reservation policy is depicted in Figure 7. By allowing each user to reserve the successfully accessed chunks for a duration of time inversely proportional to amount of bandwidth occupied, the problem of outage due to unavailability of idle chunks is avoided. Consequently, the guaranteed user throughput does not deteriorate when the offered load is increased as long as the threshold parameter is set so as to enable the cell-edge users to meet their minimum SINR targets (see Figure 7a). Interestingly, when the average traffic load in the system is less than $40 \mathrm{Mbps} / \mathrm{cell}$, the performance of dynamic chunk allocation using $\mathrm{BB}$ protocol is the same regardless of whether or not the fair scheduling policy is applied. This is because at such offered loads, the user buffer got emptied periodically and the users released the chunks they had reserved. Therefore, the chunks were still available to users who entered the network at a later point in time.

When the offered load in the system is increased to 40 $\mathrm{Mbps} / \mathrm{cell}$, the BB protocol with fair reservation achieves up to $14 \%$ higher guaranteed user throughput (Figure 7 a) together with a $13 \%$ increase in median system throughput (Figure 7d) compared to the system using static chunk allocation. Moreover, it can be observed that the dynamic chunk allocation with $\mathrm{BB}$ continues to outperform the static resource partitioning approach, both in terms of guaranteed user throughput and median system throughput until an offered load of 100 $\mathrm{Mbps} / \mathrm{cell}$ is imposed on the system. This demonstrates that the $\mathrm{BB}$ protocol with fair reservation policy allows the spectrum to be shared more flexibly among competing users and across APs in the network, thereby improving the spectral efficiency. By contrast, the static resource allocation leaves some of the chunks unoccupied in the APs where the instantaneous offered load is low while it is unable to cope with high instantaneous offered load in other APs. It should be noted that the difference between the guaranteed user throughput obtained using the $\mathrm{BB}$ protocol with fair reservation and that obtained using resource partitioning decreases as the offered load increases and eventually the static resource partitioning approach slightly exceeds the performance of the former. This is attributed to the fact that the number of chunks that remain idle decrease with an increase in the offered load, eventually leading to no idle chunks within the bandwidth assigned to the tagged AP. By contrast, with the BB signalling approach, some of the chunks remain idle for up to 3 frames when they are released in order to conform to the fair reservation policy. Furthermore, the results obtained using the full buffer traffic model had established that a reuse factor of 3 is an ideal reuse factor at the cell-edge (see Figure 5b). Due to the above two factors, the performance of the BB protocol with fair reservation suffers slightly when the offered load gradually approaches the highest value (Figure 7a). Likewise, the median system throughput obtained with the BB protocol degrades when the fair reservation policy is applied, assuming that the same threshold is used both with and without fair policy (see Figures $6 \mathrm{~d}$ vs. $7 \mathrm{~d}$ ). This is partly attributed to the fact that the chunk is released after reaching the reservation threshold and partly attributed to the fact that some chunks must remain unused in the tagged cell to avoid causing detrimental CCI to the celledge users served in neighbouring cell.

In summary, the results have established that the $\mathrm{BB}$ protocol with fair reservation policy provides a scalable mechanism to flexibly share the available chunks in the system by adjusting the threshold parameter to strike a desired balance between the conflicting goals of enhancing spectral efficiency against improving user satisfaction in the system. This self-organising property is particularly important for deployment scenarios such as the data access networks in aircraft cabins where the density of users is particularly high and the network potentially needs to cater for a wide variety of traffic classes.

\section{Conclusions}

This article addressed self-organising allocation of chunks in an optical wireless OFDMA-TDD network deployed inside an aircraft cabin using the BB protocol. The performance of the proposed $\mathrm{BB}$ approach was compared against that of a chunk allocation approach where interference is avoided through the use of cluster based static resource partitioning. Compared to static resource partitioning using a reuse factor of 3 , the $\mathrm{BB}$ approach improves the median system throughput by $17 \%$ while maintaining an equivalent throughput at the cell-edge, assuming a full buffer traffic model. Moreover, it was demonstrated that the BB specific threshold 


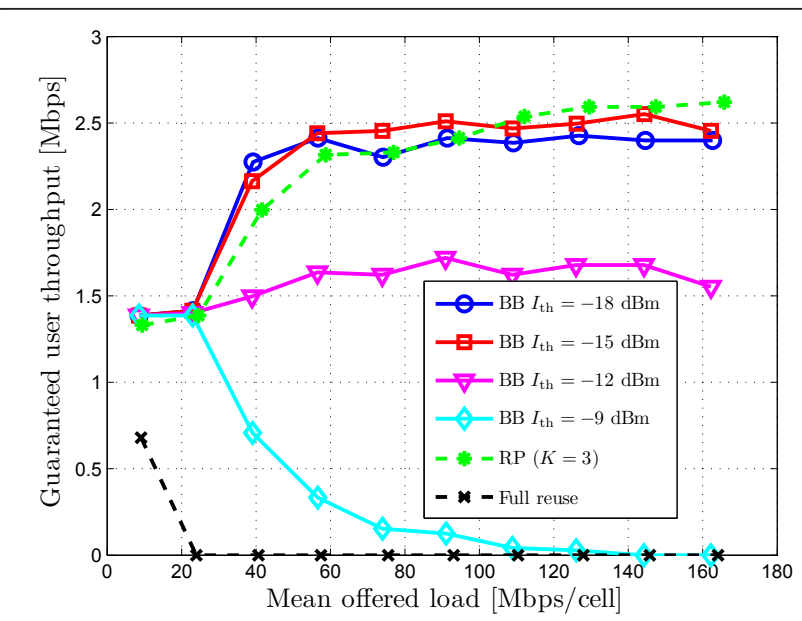

(a) Guaranteed user throughput

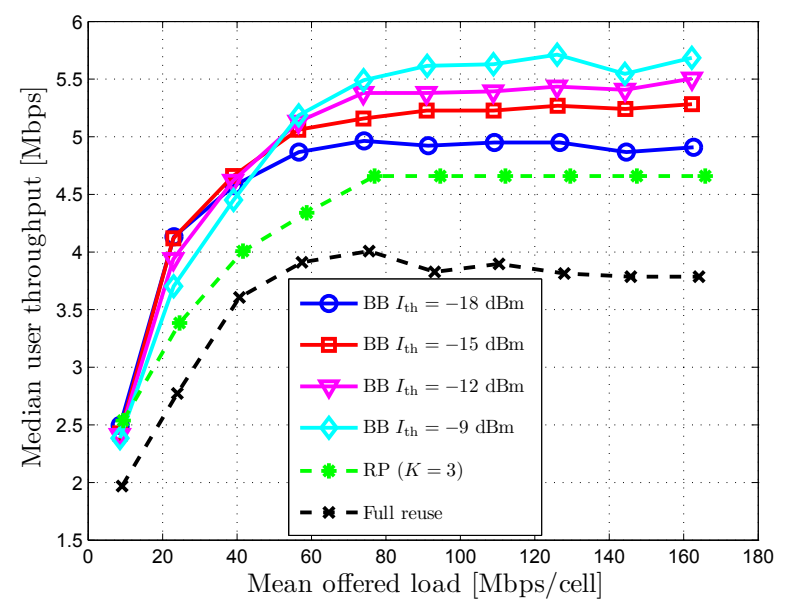

(c) Median user throughput

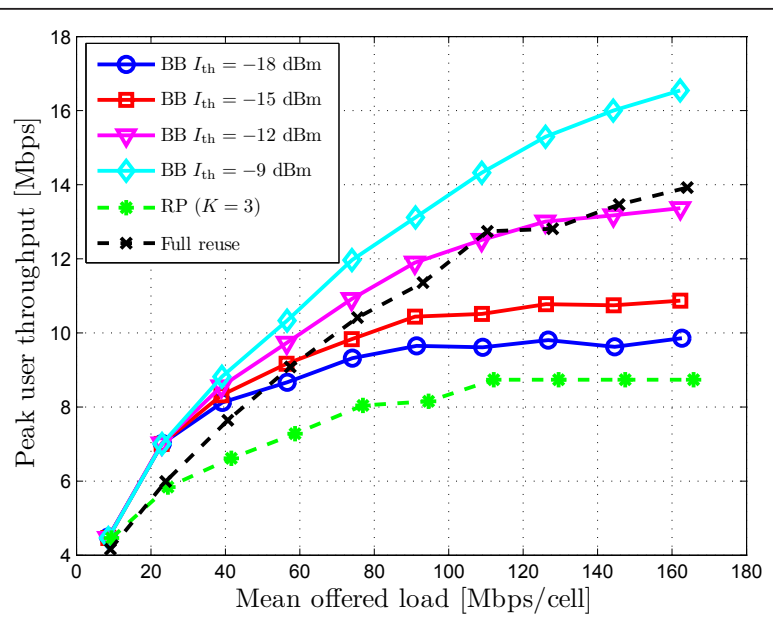

(b) Peak user throughput

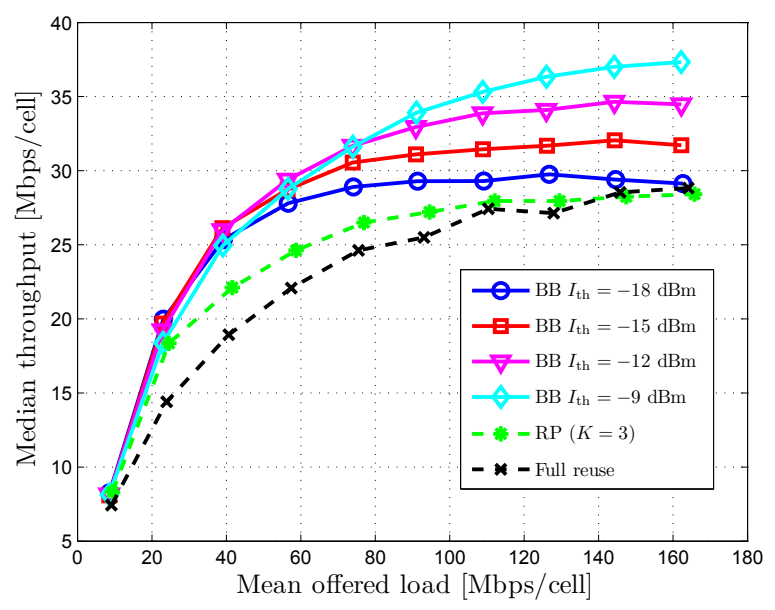

(d) Median cell throughput

Figure 7 Comparison of the performance of chunk allocation using BB signalling where the successfully accessed chunks are reserved for a period of time inversely proportional to the amount of bandwidth available to the user.

parameter can be adjusted to trade off cell-edge user throughput for aggregate system throughput and vice versa. In this context, either a median system throughput of $46 \mathrm{Mbps} / \mathrm{cell}$ or a cell-edge user throughput of 2.6 Mbps was demonstrated to be feasible with the $\mathrm{BB}$ protocol using a total system bandwidth of $20 \mathrm{MHz}$. A heuristic for fair reservation of chunks is proposed that mitigates the problem of outage that arises when the users with heavy traffic demand reserve the available chunks, leaving no chunks available to the new users trying to access the network. The results demonstrate that the proposed method outperforms the static cluster-based resource partitioning approach by $14 \%$ both in terms of median system throughput and guaranteed user throughput. Since the decision whether or not to reuse a chunk is based on comparing the received $\mathrm{BB}$ power against a threshold value, the network can be dynamically reconfigured to satisfy the guaranteed data rates or BER of different service classes simply by adjusting the threshold parameter. This self-organising property is particularly deemed important in optical wireless networks deployed inside an aircraft cabin, which is characterised by high user density and heterogeneous traffic demands.

\section{Acknowledgements}

We acknowledge partial support from the German Federal Ministry of Economics and Technology (BMWi) under grant 20K0806G (SINTEG), and partial support from the Engineering and Physical Sciences Research Council (EPSRC) under grant EP//013539/1 (Dynamic Adaptation in Heterogeneous Multicore Embedded Processors).

\section{Author details}

'School of Engineering and Science, Jacobs University Bremen, 28759, Bremen, Germany ${ }^{2}$ Institute for Digital Communications, University of Edinburgh, EH9 3JL, Edinburgh, UK 


\section{Competing interests}

The authors declare that they have no competing interests.

Received: 30 June 2011 Accepted: 4 April 2012 Published: 4 April 2012

\section{References}

1. M Wolf, D Kress, Short-range wireless infrared transmission: the link budget compared to RF. IEEE Wirel Commun Mag. 10(2), 8-14 (2003)

2. T Komine, M Nakagawa, Fundamental analysis for visible-light communication system using LED lights. IEEE Trans Cons Electron. 50(1), 100-107 (2004)

3. M Miki, E Asayama, T Hiroyasu, Visible-light communication using visiblelight communication technology, in Proceeding of the IEEE Conference on Cybernetics and Intelligence Systems (CIS 06), Bangkok, Thailand, 1-6 (7-9 June 2006)

4. D O'Brien, G Parry, P Stavrinou, Optical hotspots speed up wireless communication. Nature Photon. 1, 245-247 (2007)

5. J Armstrong, OFDM for optical communications. J Lightwave Technol. 27(3), 189-204 (2009)

6. J Vućić, C Kottke, S Nerreter, KD Langer, JW Walewski, 513 Mbit/s visible light communications link based on DMT-modulation of a white LED. J Lightwave Technol. 28(24), 3512-3518 (2010)

7. IEEE Standard 802.15.7, IEEE Standard for Local and Metropolitan Area Networks 15.7: PHY and MAC Standard for Shortrange Wireless Optical Communication Using Visible Light. IEEE Std. IEEE Standard 802.15.7

8. H Elgala, R Mesleh, H Haas, An LED model for intensity-modulated optical communication systems. IEEE Photon Technol Lett, (22)1041-1135 (2010)

9. S Dimitrov, S Sinanovic, H Haas, Clipping noise in OFDM-based optical wireless communication systems. IEEE Trans Commun (IEEE TCOM), (99) $1-10$ (2012)

10. GW Marsh, JM Kahn, Channel reuse strategies for indoor infrared wireless communications. IEEE Trans Commun. 45(10), 1280-1290 (1997)

11. S Dimitrov, R Mesleh, H Haas, M Cappitelli, M Olbert, E Bassow, On the SIR of a cellular infrared optical wireless system for an aircraft. IEEE J Sel Areas Commun (IEEE JSAC). 27(9), 1623-1638 (2009)

12. Y Argyropoulos, S Jordan, SPR Kumar, Dynamic channel allocation in interference-limited cellular systems with uneven traffic distribution. IEEE Trans Veh Technol. 48(1), 224-232 (1999)

13. K-D Langer, J Vucic, C Kottke, L Fernandez, K Habel, A Paraskevopoulos, M Wendl, $\vee$ Markov, Exploring the potentials of optical-wireless communication using white LEDs, in Proc of the International Conference on Transparent Optical Networks (ICTON), Stockholm, Sweden, 1-6 (26-30 June 2011)

14. H Uchiyama, M Yoshino, H Saito, M Nakagawa, S Haruyama, T Kakehashi, N Nagamoto, Photogrammetric system using visible light communications, in Proc of Annual Conference of IEEE Industrial Electronics (IECON), Orlando, USA, 1771-1776 (10-13 Nov 2008)

15. TYH Okada, K Masuda, M Katayama, Successive interference cancellation for hierarchical parallel optical wireless communication systems, in Proc of AsiaPacific Conference on Communications, Perth, USA, 788-792 (25-28 Oct 2005)

16. KIX Lin, K Hirohashi, High-speed full-duplex multiaccess system for leds based wireless communications using visible light, in Proc of the International Symposium on Optical Engineering and Photonic Technology (OEPT), Orlando, Florida, USA, 1-5 (10-13 July 2009)

17. P Omiyi, H Haas, G Auer, Analysis of TDD cellular interference mitigation using busy-bursts. IEEE Trans Wirel Commun. 6(7), 2721-2731 (2007)

18. B Ghimire, G Auer, H Haas, Busy bursts for trading-off throughput and fairness in cellular OFDMA-TDD. Eurasip J Wirel Commun Netw. 2009, 14. Article ID 462396, (2009)

19. McLaughlin S, Haas H, (eds.), Next Generation Mobile Access Technologies: Implementing TDD (Cambridge University Press, Cambridge, 2008). ISBN: 13:9780521826228

20. H Elgala, R Mesleh, H Haas, Predistortion in Optical Wireless Transmission Using OFDM, in Proc of the IEEE 9th International Conference on Hybrid Intelligent Systems (HIS), Shenyang Liaoning, China, 184-189 (12-14 Aug 2009)

21. G Auer, S Videv, B Ghimire, H Haas, Contention free inter-cellular slot reservation. IEEE Commun Lett. 13(5), 318-320 (2009)

22. http://catalog.osramos.com. Osram Opto Semiconductors, SFH 4730, Lead (Pb) Free Product-RoHS Comliant, Retrieved Mar. 25, 2011
23. http://sales.hamamatsu.com/assets/pdf/parts $\mathbf{S} / \mathbf{S 6 8 0 1}$ etc.pdf. Hamamatsu Photonics K.K, Datasheet: Si PIN photodiode S6801/S6898 series, Retrieved Oct. 18, 2010. Sept 2008

24. B Ghimire, G Auer, $\mathrm{H}$ Haas, Heuristic thresholds for busy burst signalling in a decentralised coordinated multipoint network, in Proc of the 73rd IEEE Vehicular Technology Conference (VTC), Budapest, Hungary, 1-5 (15-19 May 2011)

doi:10.1186/1687-1499-2012-131

Cite this article as: Ghimire and Haas: Self-organising interference coordination in optical wireless networks. EURASIP Journal on Wireless Communications

and Networking 2012 2012:131.

\section{Submit your manuscript to a SpringerOpen ${ }^{\circ}$ journal and benefit from:}

- Convenient online submission

- Rigorous peer review

- Immediate publication on acceptance

- Open access: articles freely available online

- High visibility within the field

- Retaining the copyright to your article

Submit your next manuscript at $\gg$ springeropen.com 